\title{
Critical Tests of Unintegrated Gluon Distributions
}

\author{
H. Jung ${ }^{1}$, A.V. Kotikov ${ }^{2}$, A.V. Lipatov ${ }^{3}$ and N.P. Zotov ${ }^{3}$ \\ 1- DESY, Hamburg, Germany \\ 2- BLTHPH - JINR, Dubna, Russia \\ 3- SINP - MSU, Moscow, Russia
}

\begin{abstract}
We use the unintegrated Parton Density Functions of the gluon obtained from a fit to measurements of the structure functions $F_{2}\left(x, Q^{2}\right)$ and $F_{2}^{c}\left(x, Q^{2}\right)$ at HERA to describe the experimental data for $F_{2}^{b}\left(x, Q^{2}\right), F_{L}\left(x, Q^{2}\right)$ and $F_{L}$ at fixed $W$.
\end{abstract}

\section{Introduction}

The purpose of the present investigation is to study the longitudinal structure function (SF) $F_{L}\left(x, Q^{2}\right)$ as well as the charm and beauty contributions to the proton $\mathrm{SF} F_{2}\left(x, Q^{2}\right)$ using the $k_{T}$-factorization approach of QCD [2]. The SF $F_{L}\left(x, Q^{2}\right)$ is directly connected to the gluon density in the proton. Only in the naive quark-parton-model $F_{L}\left(x, Q^{2}\right)=0$, and becomes non-zero in pQCD. However the pQCD leads to controversal results still. It was shown recently [3], that the $F_{L}$ experimental data from HERA seem to be inconsistent with some of the NLO predictions (in particular the MRST one) at small $x$. BFKL effects significantly improve the description of the low $x$ data when compared to a standard NLO $\overline{M S}$-scheme global fit. The NNLO global fit becomes better when taking into account higher order terms involving powers of $\ln (1 / x)$. It means, that we need a resummation procedure.

On the other hand it is known, that the BFKL effects are taken into account from the very beginning in the $k_{T}$-factorization approach [2], which is based on the BFKL [4] or CCFM [5] evolution equations summing up the large logarithmic terms proportional to $\ln (1 / x)$ or $\ln (1 /(1-x))$ in the LLA. Some applications of the $k_{T}$-factorization approach were shown in Refs. [6]. In the framework of $k_{T}$-factorization the study of the longitudinal SF $F_{L}$ began already ten years ago [7], where the small $x$ asymptotics of $F_{L}$ has been evaluated, using the BFKL results. Since we want to analyze the SF data in a broader range at small $x$ we use a more phenomenological approach in our analyses of $F_{2}$ and $F_{L}$ data $[8,9]$. Using the $k_{T}$-factorization approach for the description of different $\mathrm{SF}$ at small $x$ we hope to obtain additional information (or restrictions), in particular, about one of the main ingradient of $k_{T}$-factorization approach - the unintegrated gluon distribution (UGD)

In the $k_{T}$-factorization the $\mathrm{SF} F_{2, L}\left(x, Q^{2}\right)$ are driven at small $x$ primarily by gluons and are related in the following way to the UGD $x \mathcal{A}\left(x, \mathbf{k}_{T}^{2}, \mu^{2}\right)$

$$
F_{2, L}\left(x, Q^{2}\right)=\int_{x}^{1} \frac{d z}{z} \int^{Q^{2}} d k_{T}^{2} \sum_{i=u, d, s, c} e_{i}^{2} \hat{C}_{2, L}^{g}\left(x / z, Q^{2}, m_{i}^{2}, k_{T}^{2}\right) x \mathcal{A}\left(x, \mathbf{k}_{T}^{2}, \mu^{2}\right) .
$$

The functions $\hat{C}_{2, L}^{g}\left(x, Q^{2}, m_{i}^{2}, k_{T}^{2}\right)$ can be regarded as SF of the off-shell gluons with virtuality $k_{T}^{2}$ (hereafter we call them hard structure functions ). They are described by the sum of the quark box (and crossed box) diagram contribution to the photon-gluon interaction.

To apply Eq.(1) for SF at low $Q^{2}$ we change the low $Q^{2}$ asymptotics of the QCD coupling constant within hard structure functions. We have used the so called "freezing" procedure 
in the "soft" form, when the argument of the strong coupling constant is shifted from $Q^{2}$ to $Q^{2}+M^{2}$ [10]. Then $\alpha_{s}=\alpha_{s}\left(Q^{2}+M^{2}\right)$. For massless quarks $M=m_{\rho}$ and for massive ones with mass $m_{Q}, M=2 m_{Q}$.

To calculate the SF $F_{2}^{c, b}$ and $F_{L}\left(x, Q^{2}\right)$ we used the hard SF $\hat{C}_{2, L}^{g}\left(x, Q^{2}, m^{2}, k_{T}^{2}\right)$ from Ref. [9, 11] and two UGD $\mathcal{A}\left(x, \mathbf{k}_{T}^{2}, \mu^{2}\right)$ obtained in our previous paper [13]. These UGD are determined by a convolution of the non-perturbative starting distribution $\mathcal{A}_{0}(x)$ and CCFM evolution denoted by $\overline{\mathcal{A}}\left(x, \mathbf{k}_{T}^{2}, \mu^{2}\right)$ :

$$
x \mathcal{A}\left(x, \mathbf{k}_{T}^{2}, \mu^{2}\right)=\int d z \mathcal{A}_{0}(z) \frac{x}{z} \overline{\mathcal{A}}\left(\frac{x}{z}, \mathbf{k}_{T}^{2}, \mu^{2}\right),
$$

where

$$
x \mathcal{A}_{0}(x)=N x^{-B_{g}}(1-x)^{C_{g}}\left(1-D_{g} x\right) .
$$

The parameters $N, B_{g}, C_{g}, D_{g}$ of $\mathcal{A}_{0}$ were determined in the fits to $F_{2}$ and $F_{2}^{c}$ data [14, 15] independently (see [13]) Fig. 1 shows the two different UGD. The small $x$ behaviour of these UGD is very different ${ }^{\mathrm{b}}$.

To calculate the SF $F_{2}^{b}\left(x, Q^{2}\right)$ and $F_{L}\left(x, Q^{2}\right)$ we took $m_{c}=1.4 \mathrm{GeV}$ and $m_{b}=$ $4.75 \mathrm{GeV}$ and used the $m^{2}=0$ limit of the above Eq. 1 to evaluate the corresponding lightquark contributions to the $F_{L}$. Fig. 2 shows the $F_{2}^{b}$ as a function of $x$ at fixed $Q^{2}$. Fig.3 shows the $F_{L}$ as a function of $x$ at fixed $Q^{2}$. Fig. 4 shows the $\mathrm{SF} F_{L}\left(Q^{2}\right)$ at fixed $W$ compared to the H1 data [18]. It is interesting to observe, that the measured $F_{2}^{b}$ seems to prefer the UGD obtained from the fit to $F_{2}$ and is inconsitent with the one obtained from $F_{2}^{c}$. Also the measured $F_{L}$ is better described with the UGD from the $F_{2}$ fit. In summary the $k_{T}$ - factorization approach with the CCFM-evolved UGD obtained from the fits to the $F_{2}\left(x, Q^{2}\right)$ data reproduces the $\mathrm{H} 1$ data for $\mathrm{SF} F_{2}^{b}\left(x, Q^{2}\right)$,

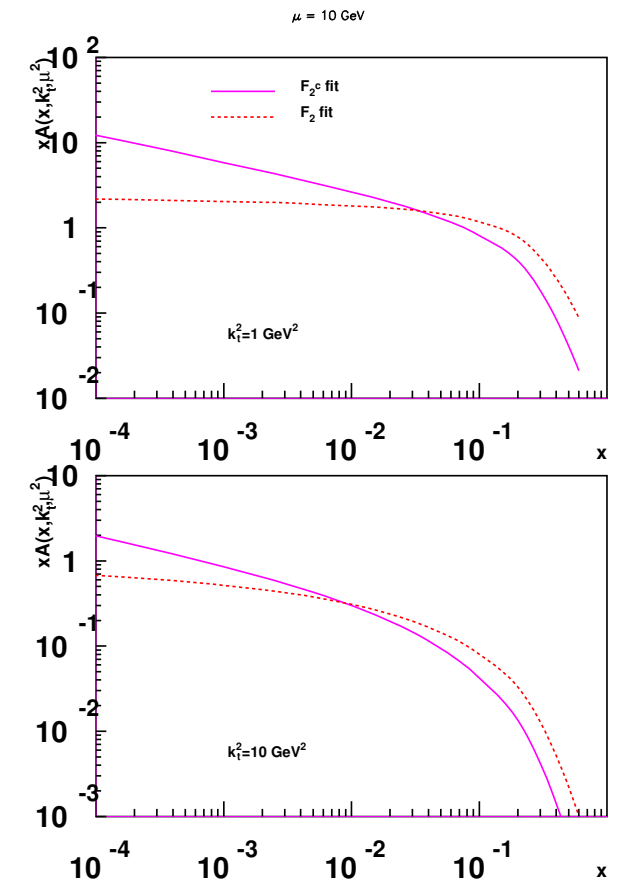
$F_{L}\left(x, Q^{2}\right)$ and $F_{L}$ at fixed $W$ (see [13]). The

Figure 1: UGD obtained in the fits to $F_{2}^{c}$ (solid curve) and $F_{2}$ (dotted curve)

UGD obtained from the fit to $F_{2}^{c}$ seems to overshoot the measured $F_{2}^{b}$ and $F_{L}$ at small $x$. New experimental data for $F_{L}\left(x, Q^{2}\right)$ but also more precise measurements of the heavy quark structure functions are very important for a precise determination of the UGD.

${ }^{a}$ There is full agreement of our results with the formulae for the photoproduction of heavy quarks from Ref. [12].

${ }^{\mathrm{b}}$ See also Ref. [16]. 

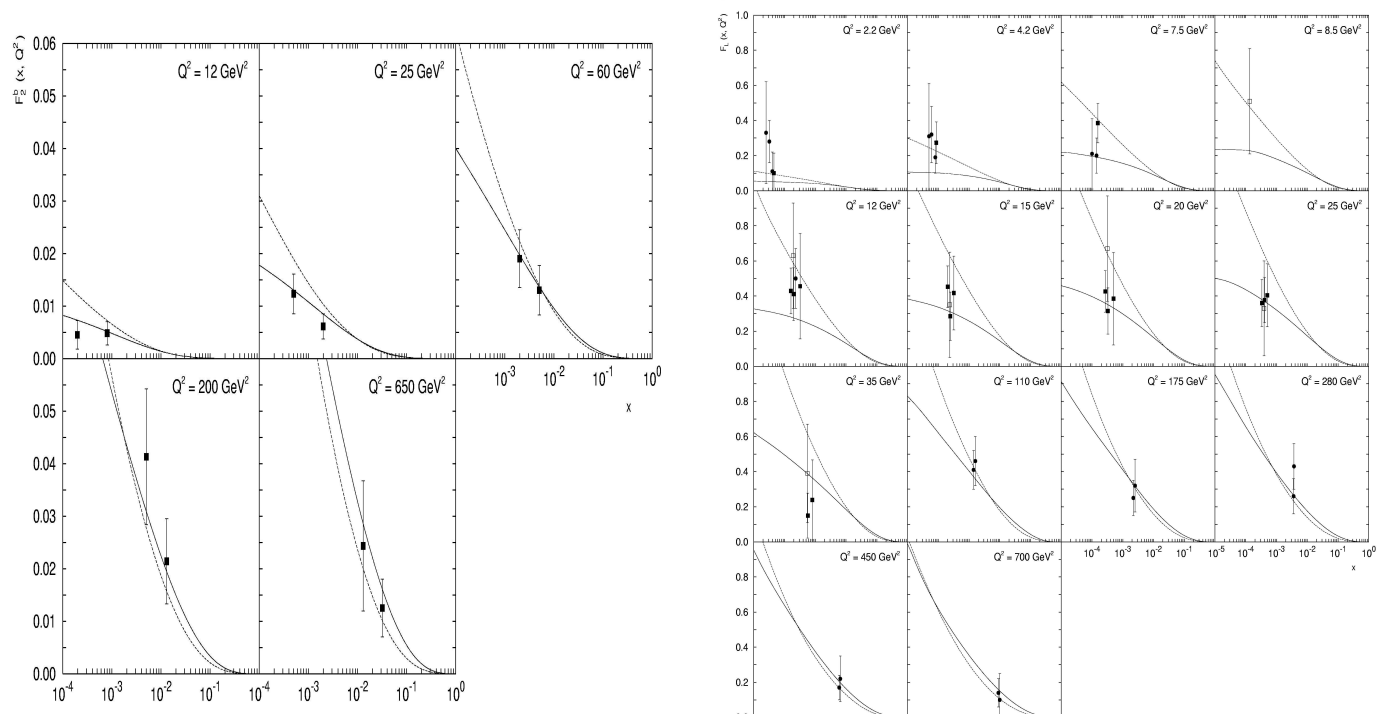

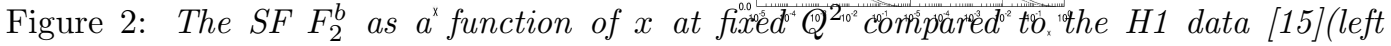
panel) The solid and dotted lines are from CCFM-evolved UGD obtained from the fits to $F_{2}\left(x, Q^{2}\right)$ and $F_{2}^{c}\left(x, Q^{2}\right)$. The $S F F_{L}$ as a function of $x$ at fixed $Q^{2}$ compared to the H1 data [14, 17](right panel) The solid and dotted lines are from CCFM-evolved UGD obtained from the fits to $F_{2}\left(x, Q^{2}\right)$ and $F_{2}^{c}\left(x, Q^{2}\right)$.

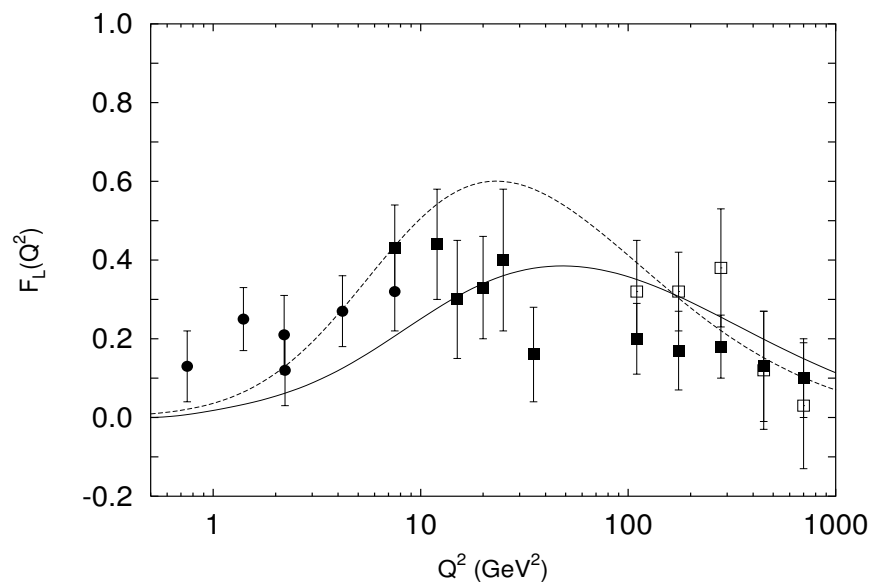

Figure 3: The $Q^{2}$ dependence of $S F F_{L}\left(Q^{2}\right)$ at fixed $W=276 \mathrm{GeV}$ compared to the H1 data [18] The solid and dotted lines are from CCFM-evolved UGD obtained from the fits to $F_{2}\left(x, Q^{2}\right)$ and $F_{2}^{c}\left(x, Q^{2}\right)$. 


\section{References}

[1] Slides: http://indico. cern. ch/contributionDisplay $\cdot$ py? contribId=55\&sessionId=8\&conf Id $=9499$

[2] S. Catani, M. Ciafaloni and F. Hautmann, Nucl. Phys. B366 135 (1991); J.C. Collins and R.K. Ellis, Nucl. Phys. B360 3 (1991);

E. Levin, M. Ryskin, Yu. Shabelski and A. Shuvaev Sov. J. Nucl. Phys. 53657 (1991).

[3] R.S. Thorne, arXiv:hep-ph/0511351; C.D. White and R.S. Thorne, Phys. Rev. D74 014002 (2006); D75 034005 (2007).

[4] L.N. Lipatov, Sov. J. Nucl. Phys. 23338 (1976); E.A. Kuraev, L.N. Lipatov and V.S. Fadin, Sov. Phys. JETP 44443 (1976); 45199 (1977); Ya.Ya. Balitzki and L.N. Lipatov, Sov. J. Nucl. Phys. 28822 (1978); L.N. Lipatov, Sov. Phys. JETP 63904 (1986).

[5] M. Ciafaloni, Nucl. Phys. B296 49 (1988);

S. Catani, F. Fiorani and G. Marchesini, Nucl. Phys. B336 18 (1995); G. Marchesini, Nucl. Phys. B445 49 (1995).

[6] Bo Andersson et al. (Small $x$ Collaboration), Eur. Phys. J. C25 77 (2002); J. Andersen et al. (Small $x$ Collaboration), Eur. Phys. J. C25 67(2002); C35 67 (2004)

[7] S. Catani and F. Hautmann, Nucl. Phys. B427 475 (1994); S. Catani, arXiv:hep-ph/9608310.

[8] B.Badelek, J.Kwiecinski and A. Stasto, Z. Phys. C74 297 (1997).

[9] A.V. Kotikov, A.V. Lipatov and N.P. Zotov, Eur. Phys. J. C26 51 (2002).

[10] N. Nikolaev and B.M. Zakharov, Z. Phys. C49 607 (1991); C53 331 (1992).

[11] A.V. Kotikov, A.V. Lipatov and N.P. Zotov, Eur. Phys. J. C27 219 (2003).

[12] S. Catani, M. Ciafaloni and F. Hautmann, Proceedings of the Workshop on Physics at HERA, Hamburg, Germany (1991), v. 2, p. 690 .

[13] H. Jung, A.V. Kotikov, A.V. Lipatov and N.P. Zotov, arXiv: hep-ph/0611093.

[14] H1 Collab., A. Adloff et al., Eur. Phys. J. C21 33 (2001).

[15] H1 Collab., A. Adloff et al., Phys. Lett. B528 199 (2002); A. Aktas et al., Eur. Phys. J. C40 349 (2005); C45 23 (2006).

[16] H. Jung, talk in HFS working group on DIS'07.

[17] H1 Collab., A. Aid et al., Phys. Lett. B393 452 (1997); N. Gogitidze, J. Phys. G28 751 (2002).

[18] E.M. Lobodzinska, Proceedings of the DIS 2003, Gatchina, St. Petersburg, Russia, p. 93. 